\title{
Primary carcinoma of the fallopian tubes: a case report
}

\section{Lalita Rajiva Mulmule*}

Dr. Mulmule Maternity and Nursing Home, Near Gopal Talkies, Rajapeth Amravati - 444605, Maharashtra, India

Received: 15 June 2016

Accepted: 02 July 2016

\section{*Correspondence:}

Dr. Lalita Rajiva Mulmule,

E-mail: drsjtiwari@gmail.com

Copyright: (c) the author(s), publisher and licensee Medip Academy. This is an open-access article distributed under the terms of the Creative Commons Attribution Non-Commercial License, which permits unrestricted non-commercial use, distribution, and reproduction in any medium, provided the original work is properly cited.

\begin{abstract}
Primary fallopian tube carcinoma (PFTC) is considered a rare and aggressive type of tumour, representing 0.14-1.8\% of the total gynaecological malignancies. About 1200 cases in all have been reported till now. It is very important to diagnose these neoplasms at an early stage as the survival rates drop dramatically with advanced stages. However, a correct diagnosis is rarely achieved preoperatively and PFTC is often mistaken for ovarian malignancies, pelvic inflammatory disease, pelvic peritonitis. We are reporting the case of a 53 year old female who presented with abnormal vaginal bleeding and discharge, and was diagnosed to have PFTC, papillary type FIGO II, only on histopathology after undergoing total abdominal hysterectomy with bilateral salphingo oophorectomy.
\end{abstract}

Keywords: PFTC, Carcinoma

\section{INTRODUCTION}

Primary fallopian tube carcinoma (PFTC) is considered a rare and aggressive type of tumour, representing 0.14 $1.8 \%$ of the total gynaecological malignancies. ${ }^{1}$

About 1200 cases in all have been reported till now.

PFTC carries 5 year survival rates of:

Stage I disease ---- 68 to $76 \%$

Stage II disease ---- 27 to $42 \%$

Stage III disease ---- 0 to $6 \%$

Hence, it is very important to diagnose these neoplasms at an early stage.

However, a correct diagnosis is rarely achieved preoperatively and in many cases, diagnosis is made only after surgery for unrelated conditions. ${ }^{2}$

PFTC is often mistaken for ovarian malignancies, pelvic inflammatory disease (PID), pelvic peritonitis.
PFTC with papillary features is the commonest histological type (90\%).

It is usually managed in the same manner as the ovarian malignancies but invariably has a worse prognosis.

\section{CASE REPORT}

A 53 year old female, 5 years postmenopausal, presented with complaints of vaginal discharge since 1 year and postmenopausal bleeding since 1 month. She had no history of lower abdominal pain, altered bowel bladder habits, bleeding per rectum, malena and haematuria. There were no chest complaints or history of fever. There was occasional backache with no significant past history. She was 5 years postmenopausal with 3 para.

She was moderately built, fairly nourished with stable vitals. There was no pallor, icterus, lymphadenopathy or edema feet.

Abdominal examination was normal. On per speculum/ vaginal examination uterus was bulky with no adnexal mass or tenderness. 
Blood investigation was normal. Ultrasonography (USG) of abdomen and pelvis did not reveal any abnormality except for bulky uterus.

Check dilatation and curettage (D and C) was done and biopsy report showed changes of chronic cervicitis without dysplasia.

Considering history, examination findings and other investigations a provisional diagnosis of dysfunctional uterine bleeding was made and the patient was advised total abdominal hysterectomy with bilateral salphingooophorectomy (TAH BSO).

Patient underwent open surgery. There was no free fluid or blood in abdomen and pelvis. The tubes on the both sides were thickened, adherent and adhesions were firm but no nodularity or tubercles were noted. Ovaries on both sides were normal. The tubo-ovarian adhesions that were, mainly omental were separated and omentum was excised.

TAH with BSO (Figure 1) was done. Post-operative period was uneventful. Histopathological findings were as follow:

1. Chronic endo cervicitis

2. Uterine $2 \times 2 \mathrm{~cm}$ adenomyomatous polyp and superficial adenomyosis seen.

3. Left ovary-small follicular cyst

4. Left fallopian tube- benign epithelium with transition to papillary adenocarcinoma (well differentiated) (stage I).

5. Right ovary - small cystic adenofibroma.

6. Right fallopian tube-epithelial lining with poorly differentiated adenocarcinoma spreading up to serosa (stage II A).

The slides were reviewed by oncologist. Post-surgery levels of CA 125/CEA were done and were normal. Final diagnosis was primary fallopian tube carcinoma (PFTC), papillary type FIGO II.

Patient was advised revision surgery after oncology opinion for pelvic, para-aortic lymph node dissection and removal of residual disease but she refused. She was put on taxol + cisplatin combination chemotherapy.

During follow-up patient did well up to one year with CA125 remaining static. After one year she developed progressive ascites and tumour marker increasing dramatically. A relook debulking surgery was advised but it was refused. Patient succumbed one and half year after surgery due to widespread metastatic disease.

\section{Interesting post script}

A month after the patient succumbed, her younger sister, age 48 years, came with complaint of breast lump and was diagnosed with Locally Advanced carcinoma Breast. She is responding well to treatment.

She was advised to go for genetic assessment of BRCA1BRCA2 mutation but she politely declined.

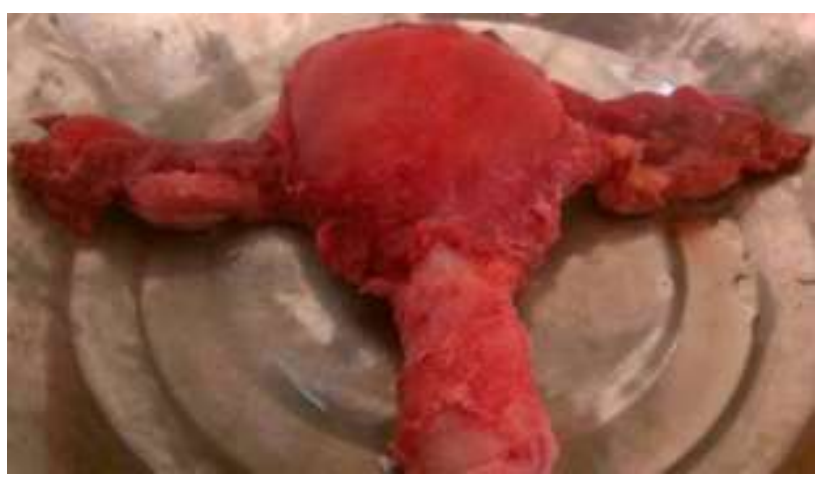

Figure 1: Specimen of total abdominal hysterectomy with bilateral salphingo oophorectomy.

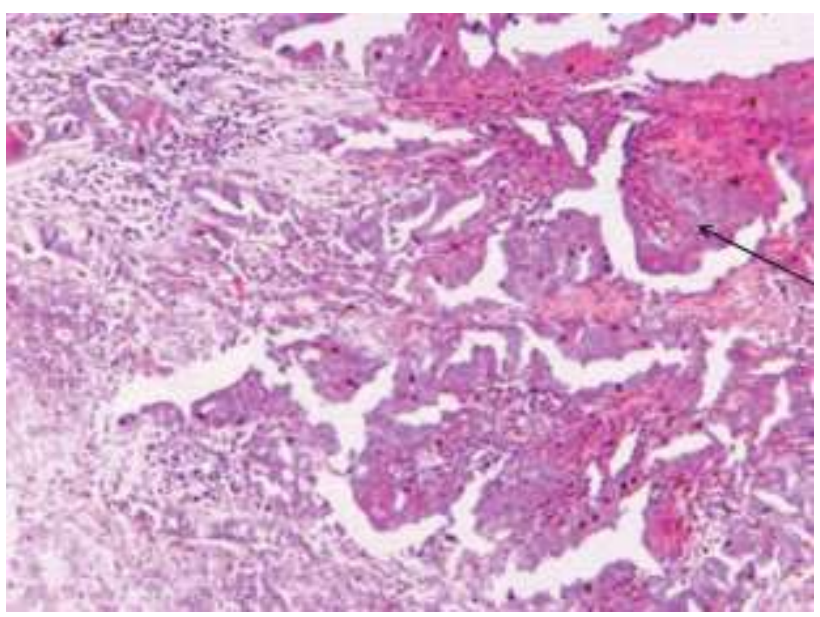

Figure 2: Fallopian tube showing papillary growth within the lumen of tube (arrow - papillary adenocarcinoma).

\section{DISCUSSION}

PFTC is the least common of all gynaecological malignancies with annual incidence of about 3.6 per million women per year. It accounts for $0.14-1.8 \%$ of all female genital malignancies.

The peak incidence is between $60-64$ years of age with mean age of incidence of 55 years (range 17-88 years). Etiology remains unclear and it could be due to hormonal, reproductive or genetic factors. ${ }^{1}$ There is a noticeable correlation with nulliparity, infertility and Pelvic inflammatory disease.

PTFC has been described in high risk breast - ovarian cancer families with germ line BRCA - I and II mutations. 
Commonest symptoms were palpable pelvic or abdominal mass, abnormal vaginal bleeding, lower abdominal pain and abnormal watery discharge.

Hydrops tubae profluens is a syndrome characterized by the relief of pain and shrinkage of the abdominal or pelvic mass by a vaginal discharge, which is caused by filling and emptying of a sub-occluded fallopian tube (seen in only $5 \%$ of patients). ${ }^{3}$

This phenomenon is explained by the partial blockage of fallopian tube caused by the malignancy.

Examination may reveal bulky uterus, tender swollen adnexa, abdominal or pelvic mass and low abdominal tenderness in early cases. Diagnosis can only be suspected clinically and because of its rarity, is rarely made correctly clinically.

However, it should be included in differential diagnosis of all cases of abnormal vaginal bleed with watery discharge and negative $\mathrm{D}$ and $\mathrm{C}$ findings, especially in $5^{\text {th }}$ and $6^{\text {th }}$ decade.

USG findings are non-specific and usually do not help as it can mimic tubo-ovarian mass, ovarian tumour or an ectopic pregnancy.

USG may show a sausage shaped mass, multi locular or multicystic mass with, cog and wheel appearance or solid mass.

CT-MRI (Computed tomography-Magnetic resonance imaging) if done may reveal associated pelvic/paraaortic lymphadenopathy, distant metastasis or ascites. ${ }^{4}$ Incidence of lymphadenopathy is more common (33\%) than ovarian malignancies. The tumour spreads by direct spread, trans-luminal spread, lymphatic spread, trans coelomic spread or haematogenous spread.

If the pre-operative diagnosis is PFTC then CA 125 should be done. It is elevated in $65 \%$ cases and will also aid in monitoring the disease recurrence. Wherever abnormal vaginal bleed is the predominant symptom, D and $\mathrm{C}$ should be done.

Diagnostic lap is rarely of help in making proper diagnosis. Surgery is the treatment of choice with TAH with BSO being the procedure of choice

Whenever diagnosis is confirmed, pre or intra operative omentectomy with pelvic and paraaortic lymphadenectomy are the necessary additional procedure.
Ascitic fluid and peritoneal biopsies should be taken as indicated in a staging procedure.

If diagnosis is made after pathological examination then a revision surgery should be offered to achieve:

1. Complete omentectomy

2. Peritoneal biopsy

3. Additional debulking if required

4. Pelvic and paraaortic lymphadenectomy

Second look laparotomy, after chemotherapy, is also a choice as in ovarian carcinoma but it rarely increases the survival rate.

Post-operative adjuvant chemotherapy is the same as used in EOC (epithelial ovarian carcinoma).

IV taxol and cisplatin are the combination of choice; Adjuvant radiotherapy has no clear role and is not used routinely

Follow up is usually done with CA 125/CEA and USG/CT scan/MRI.

\section{CONCLUSION}

PFTC is a rare entity, rarely diagnosed pre-operatively unless it is considered in the differential diagnosis of an abnormal vaginal bleeding or suspected adnexal mass in a postmenopausal woman, especially if $\mathrm{D}$ and $\mathrm{C}$ is negative.

\section{Funding: No funding sources \\ Conflict of interest: None declared \\ Ethical approval: Not required}

\section{REFERENCES}

1. Kalampokas E, Kalampokas T, Tourountous I. Primary fallopian tube carcinoma. Eur J Obstet Gynecol Reprod Biol. 2013;169(2):155-61.

2. Shaaban AM, Rezvani M. Imaging of primary fallopian tube carcinoma. Abdom Imaging. 2012;38:608-18.

3. Horng HC, Teng SW, Huang BS, et al. Primary fallopian tube cancer: domestic data and up-to-date review. Taiwan J Obstet Gynecol. 2014;53(3):28792.

4. Ma FH, Cai SQ, Qiang JW. MRI for differentiating primary fallopian tube carcinoma from epithelial ovarian cancer. J Magn Reson Imaging. 2015;42(1):42-7.

Cite this article as: Mulmule LR. Primary

carcinoma of the fallopian tubes: a case report. Int $\mathbf{J}$ Reprod Contracept Obstet Gynecol 2016;5:2869-71. 